\title{
Design and Optimization of Red-light Reflector Using Simulation Software
}

\author{
Junjia Xu, ${ }^{1}$ Chang Hong Lin, ${ }^{2}$ Yu-Ting Chen, ${ }^{2}$ Hsien-Wei Tseng, ${ }^{1}$ \\ Peng Wang, ${ }^{1}$ Cheng-Fu Yang, ${ }^{2,3^{*}}$ and Chiang-Lung Lin ${ }^{4 * *}$ \\ ${ }^{1}$ College of Artificial Intelligence, Yango University, Mawei District, Fujian 350015, China \\ ${ }^{2}$ Department of Chemical and Materials Engineering, National University of Kaohsiung, Kaohsiung 811, Taiwan \\ ${ }^{3}$ Department of Aeronautical Engineering, Chaoyang University of Technology, Taichung 413, Taiwan \\ ${ }^{4}$ Dongguan City University, Guangdong 523419, China
}

(Received March 23, 2021; accepted May 31, 2021)

Keywords: simulation, COMSOL Multiphysics, red-light reflector, optimize

The traditional distributed Bragg reflector (DBR), which is constructed with multiperiod films with alternating low and high refractive indices, has a low full width at half maximum (FWHM), but both the ripple number and ripple intensity increase outside the reflectance band. In this study, $\mathrm{Ta}_{2} \mathrm{O}_{5}$ and $\mathrm{SiO}_{2}$ films with refractive indices of 2.10 and 1.45 were used as the lowand high-refractive-index films, respectively, to design a traditional red-light DBR with a central wavelength of $7000 \mathrm{~nm}$. First, we used COMSOL Multiphysics as a tool to simulate the designed DBR. Although the designed reflector had a low FWHM value, it had high ripple intensities outside the reflectance band. Therefore, we designed a modified DBR with a structure of 15 layers and with different thicknesses of the upper and bottom layers. The modified DBR had a smaller FWHM, a higher reflectance, and smaller ripple intensities outside the reflectance band. Although the ripple intensities were lower than those of the traditional DBR, they were still higher than $20 \%$. Next, we also used the optimization function of COMSOL Multiphysics to suppress the ripple intensities, and we showed that the ripple intensities were indeed suppressed.

\section{Introduction}

Lenses and reflectors are two important optical components used for the formation of images and the creation of light distributions and beam patterns. A reflector acts as a mirror and the light can be reflected but not transmitted. ${ }^{(1)}$ Recently, many attempts have been made to design reflectors with special central wavelengths. For example, dichroic or multilayer coatings were used as dielectric films to realize interference-based reflectivity, the most prominent example being the halogen cold reflector. ${ }^{(2)}$ Its coating is dichroic and allows IR radiation to pass through the coated glass to the rear but reflects visible light out of the front of the reflector lamp. Reflectors constructed using the multilayer films can achieve high reflectance and a low full width at half maximum (FWHM). In addition, they can provide spectrally customized angular sensitivity or reflectivity properties, for example, a reflector can be designed to reflect light at a *Corresponding author: e-mail: cfyang@nuk.edu.tw ** Corresponding author: e-mail: chlulin0510@gmail.com https://doi.org/10.18494/SAM.2021.3389 
specific central wavelength. The most well-known reflector is the distributed Bragg reflector (DBR), which is also constructed using multiple layers of alternating materials with different refractive indices, and it can be used in waveguides of optical fibers.

DBRs can be fabricated by using different oxides as the multiple-layer films of different refractive indices. ${ }^{(3-5)}$ They can also be fabricated with the periodic variation of a specific characteristic (such as height) of a material waveguide, which can result in the effective refractive index of the waveguide having a periodic variation. DBRs can be applied in fiber Bragg grating technology, and are commonly used in sensing and measurements of different physical parameters, such as strain, temperature, and pressure, in aerospace, maritime, industrial engineering, military, and civil engineering applications. ${ }^{(6)}$ For example, a DBR can be used in a fiber grating interrogator, which has been integrated with a photodetector and a broad-band light source to fabricate a small and high-precision plethysmograph for continuous monitoring. ${ }^{(7)}$ Sheppard proposed the following equation to simulate the maximum reflectance ratio at a central wavelength for a designed DBR with multiple-layer films of different refractive indices: ${ }^{(8)}$

$$
R=\left[\frac{\left(n_{H}\right)^{2 P}-\left(n_{0} / n_{s}\right) \times\left(n_{L}\right)^{2 P}}{\left(n_{H}\right)^{2 P}+\left(n_{0} / n_{s}\right) \times\left(n_{L}\right)^{2 P}}\right]^{2} .
$$

The most important shortcoming of Sheppard's equation is that it can only be used to calculate the maximum reflectance ratio of a DBR, and it cannot simulate reflective spectra in a wide range, for example, from UV to IR light.

Many attempts have been made to realize DBRs with a wide range of wavelengths. For example, Yuan et al. proposed an overall transfer matrix and incorporated the refractive indexes of $\mathrm{SiO}_{2}$ and $\mathrm{Nb}_{2} \mathrm{O}_{5}$ films at different wavelengths and the thicknesses of $\mathrm{SiO}_{2}$ and $\mathrm{Nb}_{2} \mathrm{O}_{5}$ films at each layer to calculate and simulate the reflectance spectra of a blue-light DBR. They also compared the simulation and experimental results of fabricated DBRs with different pairs. ${ }^{(9)}$ The main shortcoming of the transfer matrix is that it cannot be used to optimize the reflective property of a designed DBR. Recently, many software tools have been used to design and enhance the performance of a wide variety of reflectors, because they can precisely analyze the reflection intensities and distributions of optical spectra in a wide wavelength range for an original design. COMSOL Multiphysics is very useful software for simulating and solving many scientific engineering and science problems, allowing researchers to obtain results in less time.

For example, Garg et al. used COMSOL Multiphysics to analyze and simulate the scattering contribution of spherical particles over a wide wavelength range of 75-3000 $\mu \mathrm{m}$ (or frequency range of 0.5-2.6 THz). ${ }^{(10)}$ Robles-Agudo et al. used COMSOL Multiphysics with the finite element method and Beer-Lambert law to construct a heat transfer model and analyze the absorption phenomena of laser light in $\alpha$-brass samples. ${ }^{(11)}$ Diaz de Leon-Zapata et al. used it to fully characterize silver-zinc oxide-gold (Ag-ZnO-Au) with a star-shaped geometry, which was investigated as an optical sensor. ${ }^{(12)}$ In this study, to find the efficiency of the reflective spectrum, we used the simulation tool of COMSOL Multiphysics to simulate designed DBRs with different structures. We also used COMSOL Multiphysics to design and simulate a 
modified DBR with the different thicknesses of the upper and bottom layers in comparison with a traditional DBR. The modified DBR had high reflectance and a high FWHM (defined as half the value of the wavelength range corresponding to $10-90 \%$ of the maximum reflectance). Although it had lower ripple intensities than the traditional DBR outside the reflectance band, some values were larger than $20 \%$. Therefore, we used the optimization function of COMSOL Multiphysics to reduce all the ripple intensities of the designed reflector to less than $10 \%$.

\section{Simulation Parameters}

For the DBRs, one pair of high-index $\left(\mathrm{Ta}_{2} \mathrm{O}_{5}\right.$, with refractive index of 2.127 at $\left.700 \mathrm{~nm}\right)$-lowindex $\left(\mathrm{SiO}_{2}\right.$, with refractive index of 1.455 at $\left.700 \mathrm{~nm}\right)$ bilayer films is referred to as a period. In this study, $\mathrm{Ta}_{2} \mathrm{O}_{5}$ and $\mathrm{SiO}_{2}$ films were used as the materials with high and low refractive indexes, respectively, to design red-light DBRs with different structures. The designed thicknesses of the quarter-wave $\mathrm{Ta}_{2} \mathrm{O}_{5}$ and $\mathrm{SiO}_{2}$ films were calculated using the equation $d=\lambda /(4 n)$, where $\lambda$ was $7000 \mathrm{~nm}$ (the central reflectance wavelength), $d$ was the thicknesses of the $\mathrm{Ta}_{2} \mathrm{O}_{5}$ or $\mathrm{SiO}_{2}$ films, and the $n$ values (the refractive index) were $2.127\left(\mathrm{Ta}_{2} \mathrm{O}_{5}\right)$ and $1.455\left(\mathrm{SiO}_{2}\right)$ at $\lambda(7000 \mathrm{~nm})$. Thus, the calculated thicknesses $(\lambda / 4)$ of the $\mathrm{Ta}_{2} \mathrm{O}_{5}$ and $\mathrm{SiO}_{2}$ films were 82.26 and $120.25 \mathrm{~nm}$, respectively. COMSOL Multiphysics was used to simulate DBRs with different pairs and structures.

Figure 1(a) shows a schematic diagram of the multilayer modified red-light DBR with different pairs of layers, and Fig. 1(b) shows the simulation results for DBRs with seven and 17 pairs of $0.5 \mathrm{~L}$ (low-index material $\mathrm{L}, \mathrm{SiO}_{2}$ ) $-0.5 \mathrm{H}$ (high-index material $\mathrm{H}, \mathrm{Ta}_{2} \mathrm{O}_{5}$ ) and the lowest layer of $1.3 \mathrm{~L}$. The two results showed that as the period of the designed DBRs increased from seven to 17 pairs, the maximum reflectance increased from $97.9 \%$ to approximately $100 \%$, and the FWHM decreased from 75 to $10 \mathrm{~nm}$. The DBR with 17 pairs produced a nearly square wave,

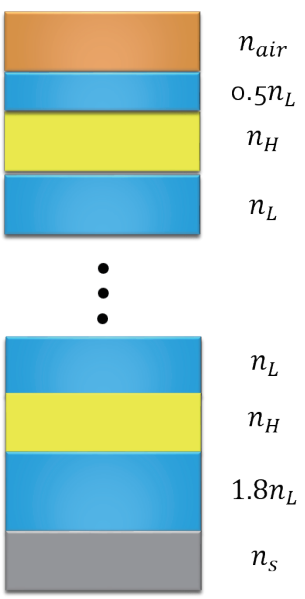

(a)

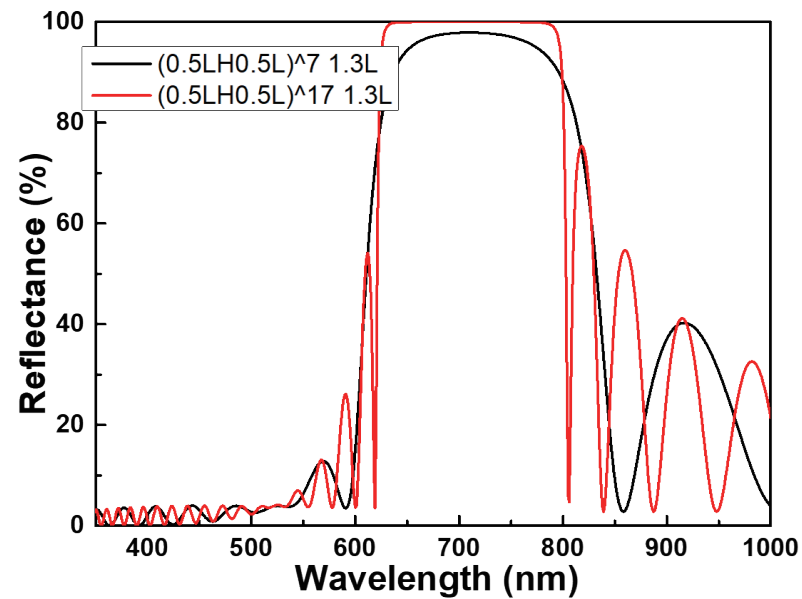

(b)

Fig. 1. (Color online) (a) Schematic diagram of a multilayer red-light DBR and (b) simulation results for designed red-light DBRs with seven and 17 pairs. 
but both the ripple number and ripple intensity outside the reflectance band increased with the period number. Two disadvantages of increasing the period of the DBR are the increased film deposition time and the increased use of source materials, increasing the fabrication cost of the DBRs. These results suggest that increasing the number of periods is not a good method to fabricate DBRs with higher performance.

Figure 2(a) shows a schematic diagram of a modified multilayer red-light DBR with a structure of $1.5 \mathrm{~L}-$ three pairs of $(0.5 \mathrm{~L}, 1 \mathrm{H}, 0.5 \mathrm{~L})-2.5 \mathrm{~L}$. The lower $2.5 \mathrm{~L}$ layer was combined with the first $0.5 \mathrm{~L}$ layer to form a $3 \mathrm{~L}$ layer, the upper $0.5 \mathrm{~L}$ was combined with the lower $0.5 \mathrm{~L}$ layer, and the top $1.5 \mathrm{~L}$ layer was combined with the final $0.5 \mathrm{~L}$ to form a $2 \mathrm{~L}$ layer. Thus, the reflector had seven layers. Figure 2(b) shows the simulation results of the modified DBR. As shown in Fig. 2(b), because the designed DBR had only three pairs, the reflectance was about $80 \%$ and the FWHM was approximately $120 \mathrm{~nm}$, which was unsatisfactory for practical application. Thus, the modified design for the DBR reduced the ripple number and the ripple intensities outside the reflectance band. The results in Figs. 1 and 2 suggest that to design a DBR with the required performance, the multilayer films should have a minimum number of layers.

The schematic diagram of another modified red-light DBR with the multilayer structure of $1.5 \mathrm{~L}-$ seven pairs of $(0.5 \mathrm{~L}, \mathrm{H}, 0.5 \mathrm{~L})-2.5 \mathrm{~L}$ is shown in Fig. 3(a), which has a structure of 15 layers. As shown in Fig. 3(a), we used the different thicknesses of the top (1.5L) and bottom (2.5L) layers to design the modified DBR. The modified top and bottom layers were used to suppress the ripple intensities outside the reflectance band, and the simulation result for the designed modified DBR is shown in Fig. 3(b). The reason for using 15 layers was that we did not want to design a DBR with too many layers, which would have increased the deposition time and manufacturing cost of the DBR. We also found that when we used 15 layers to optimize the reflectance of the modified red-light DBR, we obtained considerable reflectivity, where the optimization process to obtain the optimum structure of the red-light DBR is discussed in Sect. 3 .

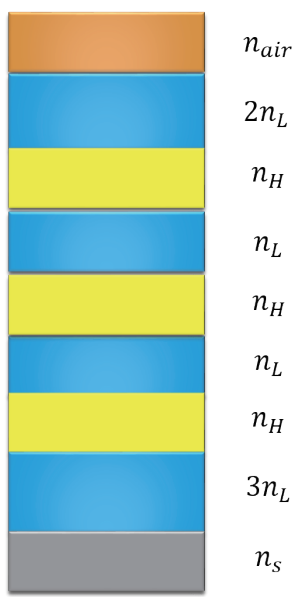

(a)

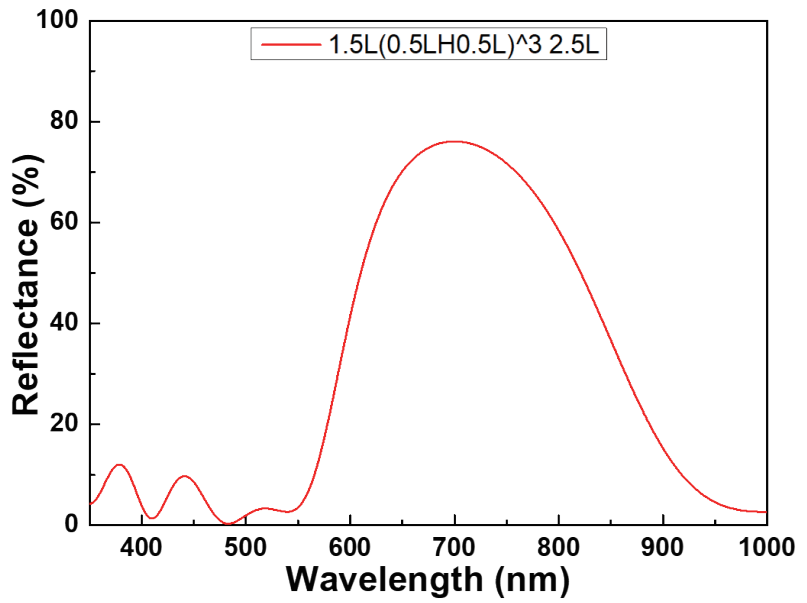

(b)

Fig. 2. (Color online) (a) Schematic diagram of a red-light DBR with three pairs and (b) simulation results for the designed DBR with eight layers. 


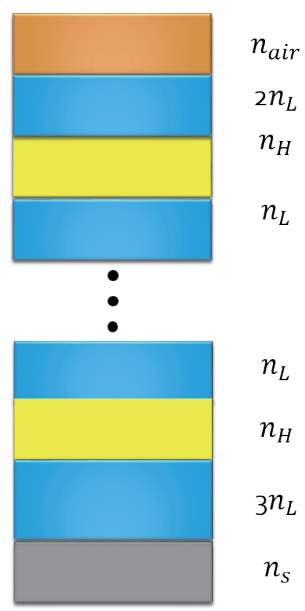

(a)

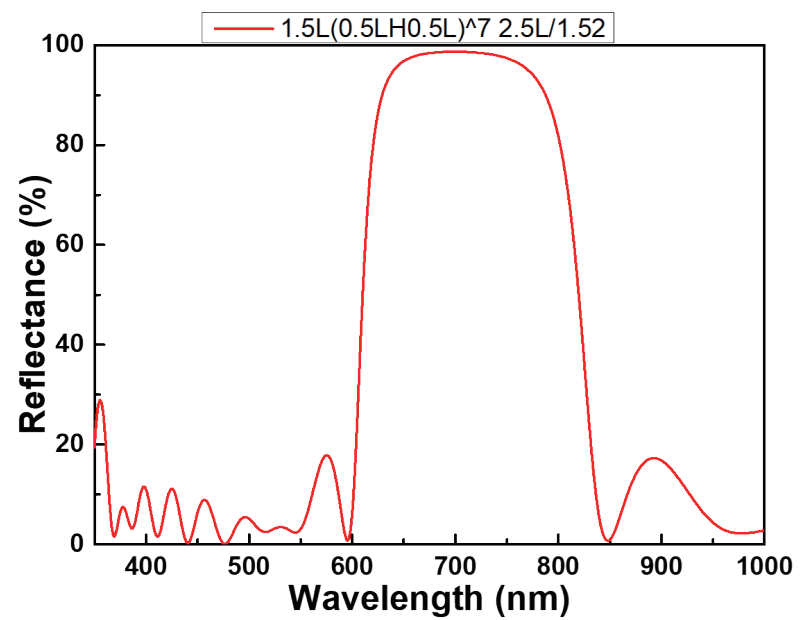

(b)

Fig. 3. (Color online) (a) Schematic diagram of a red-light DBR with seven pairs and (b) simulation results for the designed modified DBR with 15 layers.

\section{Simulation Processes and Results}

Comparison of the result shown in Fig. 3(b) with the result for the structure of seven pairs of $(0.5 \mathrm{~L}, 1 \mathrm{H}, 0.5 \mathrm{~L})-1.3 \mathrm{~L}$ shown in Fig. 1(b) reveals that the modified red-light DBR in Fig. 3(b) has better reflective characteristics because it has lower ripple intensities outside the reflectance band and a smaller FWHM of about $25 \mathrm{~nm}$, although it has irregular ripples outside the reflectance band. COMSOL Multiphysics has an advanced function that can optimize and enhance the designed device to increase its performance upon inputting the required performance. Because we desire all the ripple intensities outside the reflectance band of the designed DBR to be smaller than $10 \%$, we use the optimization function of COMSOL Multiphysics to find the optimum design parameters of the modified DBR. After we design a device, if we input the required performance, COMSOL Multiphysics can regulate the parameters of the designed structures automatically to increase its performance. For the DBR shown in Fig. 3, first we needed to suppress the ripple intensities in the ranges of 350-620 nm and $850-1000 \mathrm{~nm}$ without suppressing the reflectance in the designed band. The process used to find optimal parameters for suppressing the ripple intensities was as follows.

The two most important parameters in optimizing the designed optical devices are the sampling space and the reflectance (or transmittance) in the designed band. First, we used $50 \mathrm{~nm}$ as the sampling space to suppress the ripple intensities, where the parameters were $350 \mathrm{~nm} \leq 10 \%$, $400 \mathrm{~nm} \leq 10 \%, 450 \mathrm{~nm} \leq 10 \%$, and so on, and the result is shown in Fig. 4(a) for the first optimization process (OP-1). The parameters used to suppress the ripple intensities were a reflectance of $\leq 10 \%$ in the range of $350-620 \mathrm{~nm}$, a reflectance of $\geq 95 \%$ in the range of $620-850 \mathrm{~nm}$, and ripple intensities of $\leq 10 \%$ in the range of $850-1000 \mathrm{~nm}$. According to the result of the first step of the optimization in Fig. 4(a), there was no suppression of the ripple intensities in the range of 350-620 nm, and the spectrum in the range of 620-1000 did not 


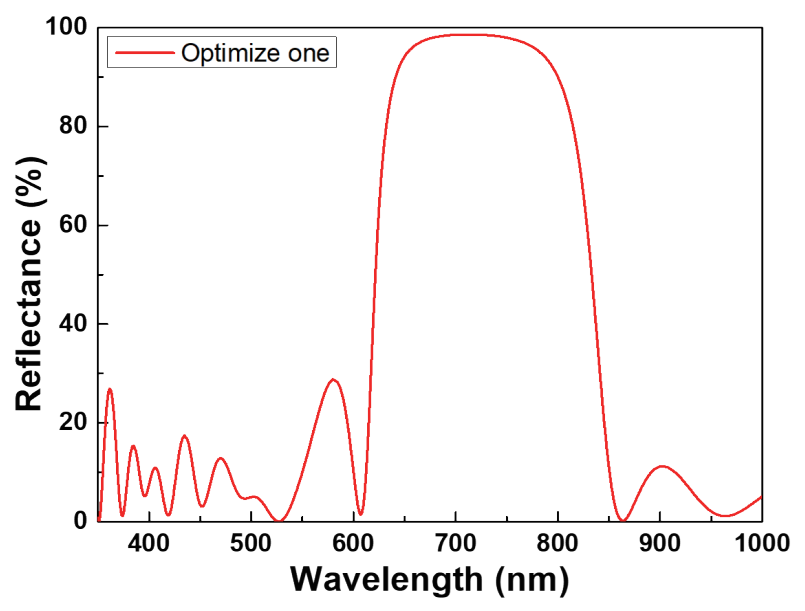

(a)

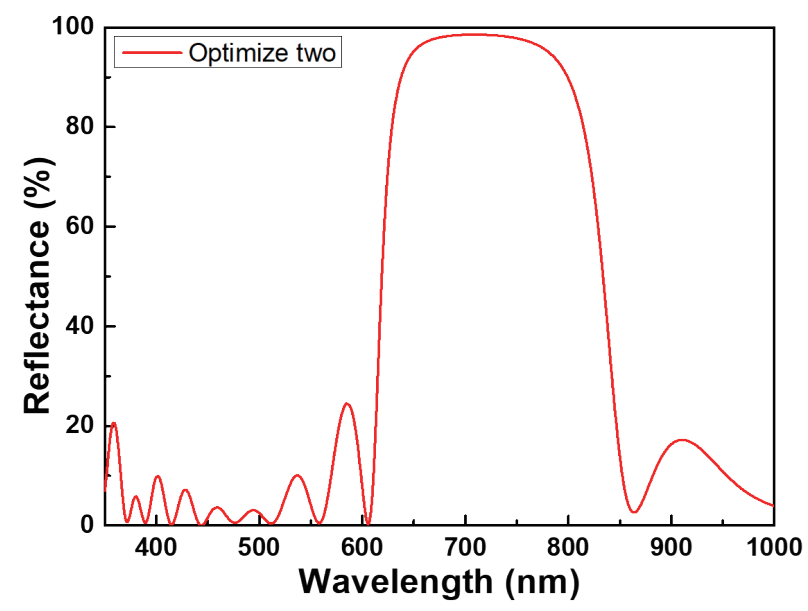

(b)

Fig. 4. (Color online) (a) OP-1 and (b) OP-2 simulation results for the designed DBR with 15 layers.

change. This is because the sampling space of $50 \mathrm{~nm}$ was too large, and the optimization process could not suppress the ripple intensities in the range of 350-620 nm. Thus, the sampling space was reduced to $25 \mathrm{~nm}$, and the result is shown in Fig. 4(b) (OP-2). However, the ripple intensities in the range of 350-620 nm only decreased slightly. The results in Fig. 4 suggest that to optimize the designed optical device and increase its performance, the sampling space needs to be smaller than $25 \mathrm{~nm}$.

To further optimize the structure, the sampling space in the range of 350-600 nm was reduced to $10 \mathrm{~nm}$, which means that the set parameters were $350 \mathrm{~nm} \leq 10 \%, 360 \mathrm{~nm} \leq 10 \%$, $370 \mathrm{~nm} \leq 10 \%$, and so on, where the result is shown in Fig. 5(a) for OP-3. The ripple intensities in the range of 350-550 nm were reduced, and the spectrum in the range of 600-1000 nm did not change. The result in Fig. 5(a) shows that if we use the sampling space of $\leq 10 \mathrm{~nm}$, then the designed optical DBR can be optimized and its performance improved. Because the ripple intensity of the peak at approximately $580 \mathrm{~nm}$ was not suppressed to below $10 \%$, a further process was required to reduce its intensity. To investigate the effect of the sampling space, the sampling space in the range of $350-600 \mathrm{~nm}$ was reduced to $2 \mathrm{~nm}$, where the result is shown in Fig. 5(b) (OP-4). The ripple intensity of the peak at approximately $580 \mathrm{~nm}$ was suppressed to below 10\%. The results in Figs. 4 and 5 suggest that the smaller the sampling space, the greater the suppression. These results also suggest that when the sampling space in the set range is changed, the properties of the spectrum outside this range do not change.

After the ripples in the range of 350-600 $\mathrm{nm}$ were suppressed, we tried to further suppress the ripple intensity at approximately $915 \mathrm{~nm}$ by setting a sampling space of $10 \mathrm{~nm}$ in the range of $850-1000 \mathrm{~nm}$, where the result is shown in Fig. 6(a) (OP-5). The spectrum in the range of 350$850 \mathrm{~nm}$ did not appear to change, and the ripple intensity was reduced slightly but its value was still larger than $15 \%$. Thus, further optimization was performed by reducing the sampling space to $2 \mathrm{~nm}$ in the range of $350-600 \mathrm{~nm}$, where the result is shown in Fig. 6(b) (OP-6). The ripple intensity of the peak at approximately $920 \mathrm{~nm}$ was suppressed to below $10 \%$. The results in 


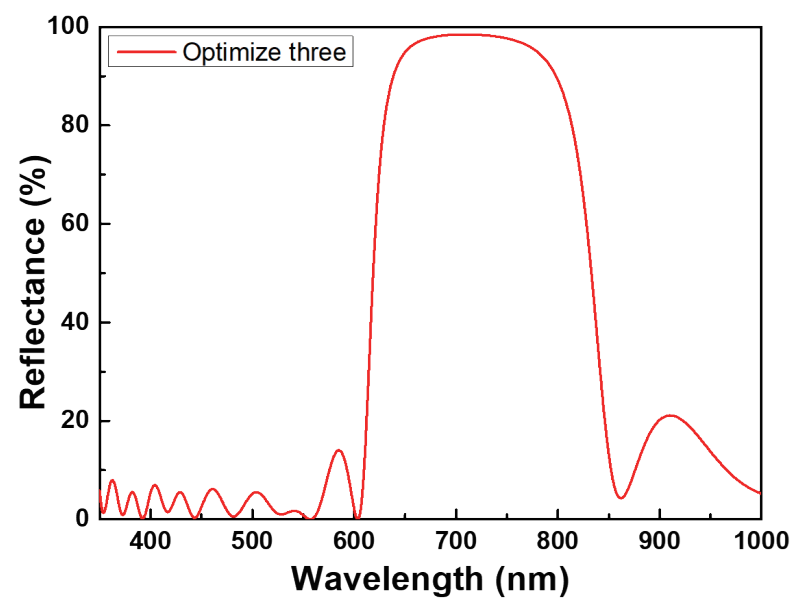

(a)

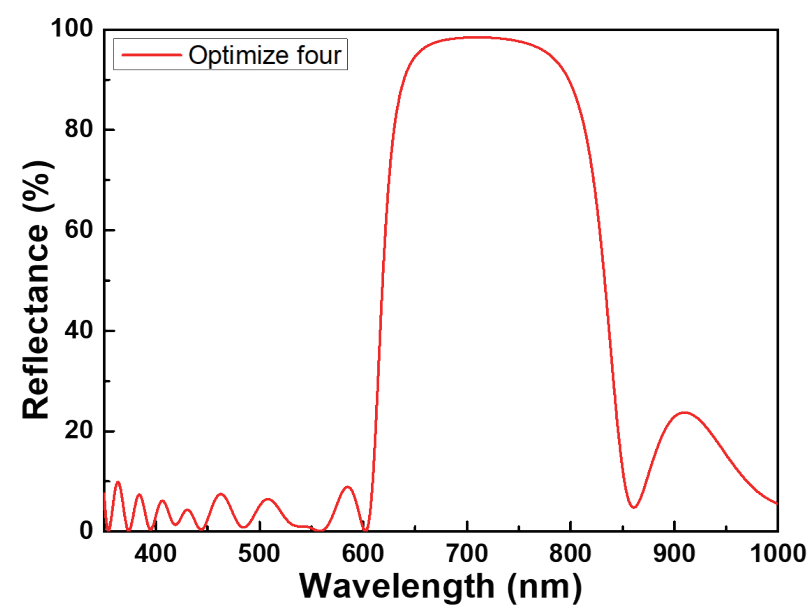

(b)

Fig. 5. (Color online) (a) OP-3 and (b) OP-4 simulation results for the designed DBR with 15 layers.

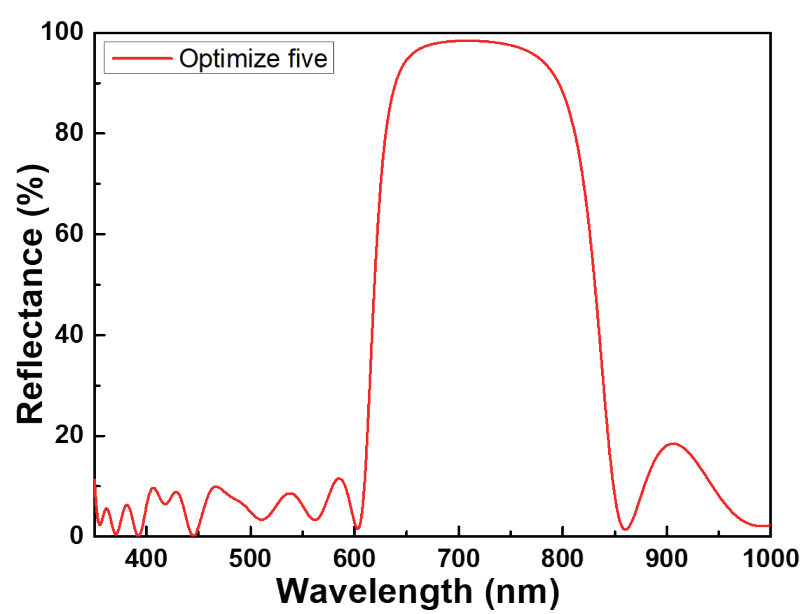

(a)

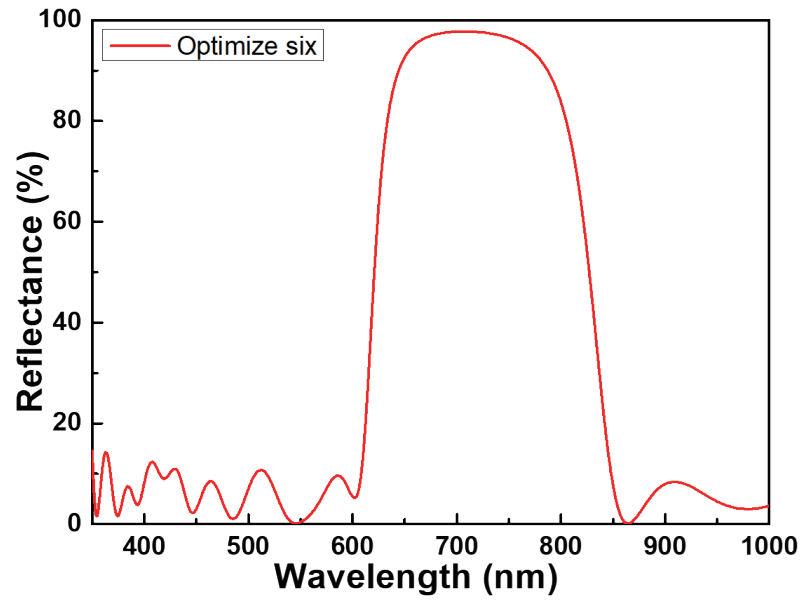

(b)

Fig. 6. (Color online) (a) OP-5 and (b) OP-6 simulation results for the designed DBR with 15 layers.

Fig. 6 also show that the smaller the sampling space, the greater the suppression of the ripple intensity.

As shown in Fig. 6(b), some of the ripple intensities in the range of 300-600 nm became higher than $10 \%$. We thus attempted to optimize the modified DBR with different parameters. The sampling space was set to $2 \mathrm{~nm}$ and only the reflectances in the wavelength ranges of $350-370 \mathrm{~nm}, 400-450 \mathrm{~nm}$, and $500-520 \mathrm{~nm}$ were set to smaller than $5 \%$. The result is shown in Fig. 7(a) (OP-7). The reflectances in these three ranges were reduced. When the sampling space was also $2 \mathrm{~nm}$, only the reflectances in wavelength ranges of 350-370 nm, 400-450 nm, and $500-520 \mathrm{~nm}$ were set to smaller than $1 \%$, and the result is shown in Fig. 7(b) (OP-8). It can be seen that the ripple intensities in the range of 300-600 $\mathrm{nm}$ were further reduced. These results also demonstrate that the two important optimization parameters are the sampling space and the 


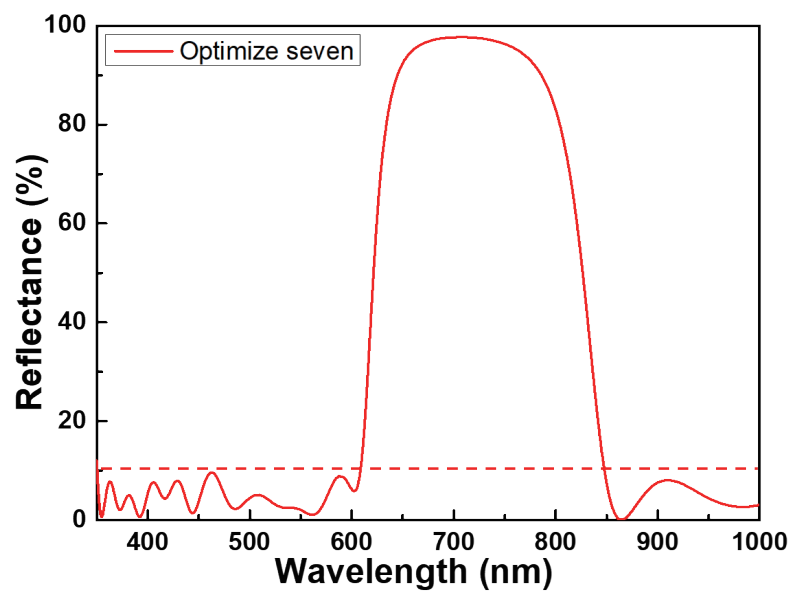

(a)

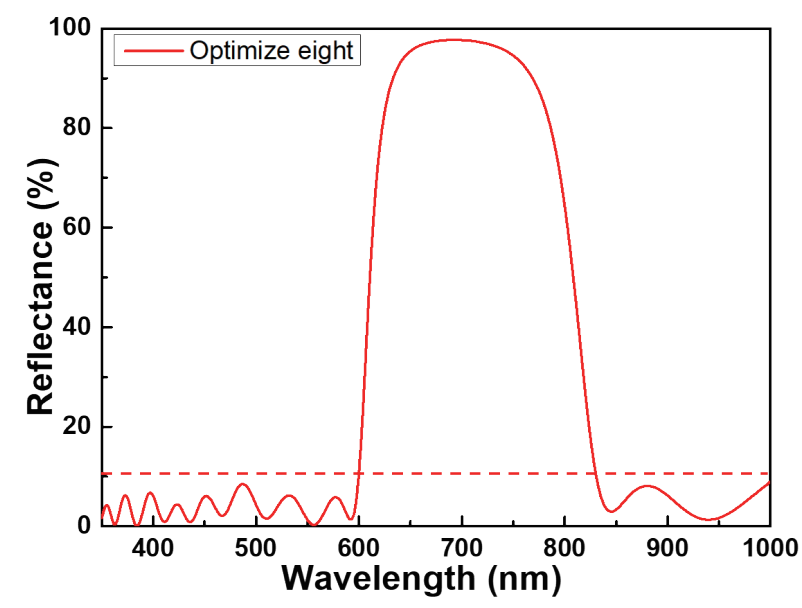

(b)

Fig. 7. (Color online) (a) OP-7 and (b) OP-8 simulation results for the designed DBR with 15 layers.

Table 1

Variations of the thickness of each film in the designed red-light reflector. OP: optimization process.

\begin{tabular}{lcrrrrrrrr}
\hline \multirow{2}{*}{ Layer } & Original & \multicolumn{1}{c}{ OP-1 } & \multicolumn{1}{c}{ OP-2 } & \multicolumn{1}{c}{ OP-3 } & \multicolumn{1}{c}{ OP-4 } & OP-5 & OP-6 & OP-7 & OP-8 \\
\cline { 2 - 9 } & Fig. 3(b) & Fig. 4(a) & Fig. 4(b) & Fig. 5(a) & Fig. 5(b) & Fig. 6(a) & Fig. 6(b) & Fig. 7(a) & Fig. 7(b) \\
\hline 1 & 240.5 & 228.92 & 226.73 & 217.00 & 217.23 & 216.81 & 212.68 & 210.51 & 212.24 \\
2 & 82.26 & 52.85 & 84.23 & 91.15 & 92.37 & 100.59 & 86.58 & 89.53 & 93.04 \\
3 & 120.25 & 172.39 & 123.65 & 129.12 & 131.36 & 129.54 & 138.42 & 135.75 & 131.05 \\
4 & 82.26 & 74.00 & 85.26 & 77.43 & 75.69 & 71.87 & 73.58 & 74.51 & 74.24 \\
5 & 120.25 & 81.50 & 122.19 & 123.74 & 124.04 & 125.3 & 124.71 & 123.84 & 120.28 \\
6 & 82.26 & 99.35 & 82.70 & 81.51 & 81.53 & 81.60 & 79.56 & 81.56 & 78.78 \\
7 & 120.25 & 151.83 & 122.14 & 124.58 & 123.78 & 124.6 & 122.12 & 124.42 & 123.51 \\
8 & 82.26 & 34.28 & 83.32 & 83.12 & 82.76 & 83.37 & 89.14 & 83.35 & 80.00 \\
9 & 120.25 & 155.18 & 122.57 & 123.69 & 123.6 & 123.20 & 120.31 & 122.27 & 122.67 \\
10 & 82.26 & 96.34 & 82.84 & 81.54 & 82.09 & 83.71 & 85.04 & 87.62 & 85.86 \\
11 & 120.25 & 61.85 & 125.13 & 124.49 & 123.03 & 131.22 & 150.1 & 151.93 & 154.07 \\
12 & 82.26 & 92.44 & 82.21 & 76.30 & 77.04 & 66.23 & 32.78 & 28.66 & 20.90 \\
13 & 120.25 & 134.48 & 124.17 & 134.42 & 134.14 & 129.17 & 153.64 & 157.97 & 156.21 \\
14 & 82.26 & 64.11 & 83.37 & 92.73 & 96.61 & 95.89 & 107.92 & 102.49 & 94.28 \\
15 & 360.75 & 375.82 & 483.55 & 490.66 & 495.64 & 516.32 & 510.34 & 516.75 & 525.00 \\
\hline Total & 1898.57 & 1875.34 & 2034.06 & 2051.48 & 2060.91 & 2079.42 & 2086.92 & 2091.16 & 2072.13 \\
\hline
\end{tabular}

reflectance value. When the sampling space and the set reflectance are set to smaller values, the optimized DBR can meet more stringent requirements.

The variations of the thickness of the 15 layers, the corresponding figure number, and the total thicknesses are shown in Table 1 for the different optimization processes. As shown in Table 1, the thicknesses of the 15 layers in the designed DBR changed slightly during the optimization processes. The total thickness of the 15-layer DBR increased from $1898.57 \mathrm{~nm}$ to 2072.13-2091.16 nm, which were the thicknesses of OP-6-OP-8. These results demonstrate that the optimization function of COMSOL Multiphysics can enhance the performance of a designed DBR with only a $10 \%$ increase in the thickness of the multilayer structure. 


\section{Conclusions}

We used COMSOL Multiphysics to design and simulate different modified DBRs with high performance. We had found that to design a DBR with a desired performance, the multilayer films should have a minimum number of layers. The designed modified DBRs had high reflectance in the required band and low ripple intensities outside the reflectance band. We found that when the optimization function of COMSOL Multiphysics was used in the simulation process, the two important optimization parameters were the sampling space and the set reflectance value. By reducing the sampling space and the set reflectance value, we were able to design a DBR that satisfied all our requirements with only a $10 \%$ increase in the thickness of the designed DBR.

\section{Acknowledgments}

This work was supported by project Nos. MOST 108-2221-E-390-005 and MOST 109-2221E-390-023.

\section{References}

1 G. W. De Young: SIAM Rev. 42 (2000) 727.

2 A. Bielawny: Adv. Opt. Technol. 8 (2019) 469.

3 T. H. Chang, T. E. Lee, N. K. Hsueh, C. H. Lin, and C. F. Yang: Micros. Technol. 24 (2018) 3941.

4 J. Liu, C. Y. Lin, W. C. Tzou, N. K. Hsueh, C. F. Yang, and Y. S. Chen: Cryst. Growth Design 18 (2018) 5426.

5 S. M. You, C. Y. You, J. J. Lin, and C. F. Yang: Functional Mater. Let. 13 (2020) 2051043.

6 J. K. Sahota, N. Gupta, and D. Dhawan: Opt. Eng. 59 (2020) 060901.

7 K. Ogawa, S. Koyama, Y. Haseda, K. Fujita, H. Ishizawa, and K. Fujimoto: Sensors 19 (2019) 3222.

8 C. J. R. Sheppard: J. Eur. Opt. Soc. Part A 4 (1995) 665.

9 Z. S. Yuan, J. M. Jhang, P. H. Yu, C. M. Jiang, Y. C. Huang, Y. L. Wu, J. J. Lin, and C. F. Yang: Vacuum 13 (2020) 109782.

10 D. Garg, A. Bandyopadhyay, and A. Sengupta: Comsol Conf. Bangalore (Bangalore, 3 October 2019).

11 M. Robles-Agudo, R. Velazquez-Hernandez, P. Duran-Arenas, and I. Rojas-Rodriguez: Comsol Conf. (Bangalore, 3 October 2019).

12 R. Diaz de Leon-Zapata, J. V. Gonzalez-Fernandez, A. B. de la Rosa-Zapata, J. E. Sanchez, G. Gonzalez, E. Flores-Garcia, and J. Gonzalez: Comsol Conf. (Bangalore, 13-14 October 2020). 